\title{
Creating Responsive Surfaces with Tailored Wettability Switching Kinetics and Reconstruction Reversibility
}

\author{
Julie A. Crowe and Jan Genzer* \\ Department of Chemical and Biomolecular Engineering, North Carolina State University, \\ Raleigh, North Carolina 27695-7905
}

Received October 10,2005; E-mail: Jan_Genzer@ncsu.edu

A responsive surface can adjust its physicochemical characteristics in response to some external stimulus, such as an electrical, chemical, or mechanical trigger. ${ }^{1}$ Examples of such surfaces include sensors that allow passage of insulin in the presence of glucose or biomimetic materials that activate a mechanical response in artificial joints. ${ }^{2}$ In most cases, the responsiveness of the surface is a result of the rearrangement of the various chemical functionalities present close to or directly at the surface. ${ }^{3-5}$ The two key parameters that define the functionality and, ultimately, the application of any responsive surface are (1) the degree of change of the surface properties after the external trigger is applied, and (2) the rate at which this change occurs. Hence, an ultimate responsive surface is one that exhibits instantaneous and measurable property variation upon application of the external stimulus. In this communication, we demonstrate that such responsive surfaces can be engineered by grafting amphiphilic moieties to a functional siloxane elastomer (SE) backbone. The combination of the high flexibility of the SE chain and the amphiphilic nature of the side groups endows these materials with exceptionally fast surface reconstruction kinetics and a high degree of repeatability.

Recently, we showed that responsive surfaces can be generated by either physical $^{6}$ or chemical ${ }^{7}$ modification of poly(vinylmethylsiloxane) (PVMS) networks. We first observed remarkably fast surface reconstruction after functionalizing the PVMS surface with carboxyl-terminated alkanes. ${ }^{7}$ To comprehend the mechanism of surface rearrangement, we chemically graft mercaptoalkanols with a variable number of the methylene spacers. We study the surface rearrangement kinetics by monitoring wettability changes using both static and dynamic contact angle measurements. To further characterize surface reconstruction in the mercaptoalkanol-modified PVMS substrates, we expose them to alternating wet and dry cycles and utilize dynamic contact angle measurements to study hysteresis in the surface reorganization. We demonstrate that increasing the number of methylene spacers in the mercaptoalkanols leads to slower reorganization kinetics and eventual loss of surface switching due to the tendency of these side groups to form semicrystalline domains at the surface. ${ }^{8-10}$

Elastomeric PVMS network substrates were synthesized by following an alkoxy-cure method as described elsewhere. ${ }^{6,7}$ The storage modulus $\left(G^{\prime}\right)$ of our networks was measured to be $\sim 8 \times$ $10^{4} \mathrm{~Pa}$ after Soxhlet extraction using toluene, followed by drying at $75{ }^{\circ} \mathrm{C}$ under $30 \mathrm{~mm} \mathrm{Hg}$ vacuum. The vinyl moieties on PVMS were subsequently saturated via $\mathrm{AIBN}$-initiated thiolene radical addition of $\mathrm{HS}\left(\mathrm{CH}_{2}\right)_{n} \mathrm{OH}(n=2,6$, and 11$) .{ }^{11}$ After the reaction, the samples (denoted as PVMS $-\mathrm{C}_{n}-\mathrm{OH}$ ) were re-extracted, dried, and stored in a low-humidity chamber. Experiments using infrared (IR) spectroscopy confirmed that the grafting density of $-\mathrm{S}\left(\mathrm{CH}_{2}\right)_{n}{ }^{-}$ $\mathrm{OH}$ was approximately equal in each sample. In Figure 1, we compare the contact angle of deionized (DI) water $\left(\theta_{\text {DIW }}\right)$ as a function of time for each mercaptoalkanol-modified PVMS surface.

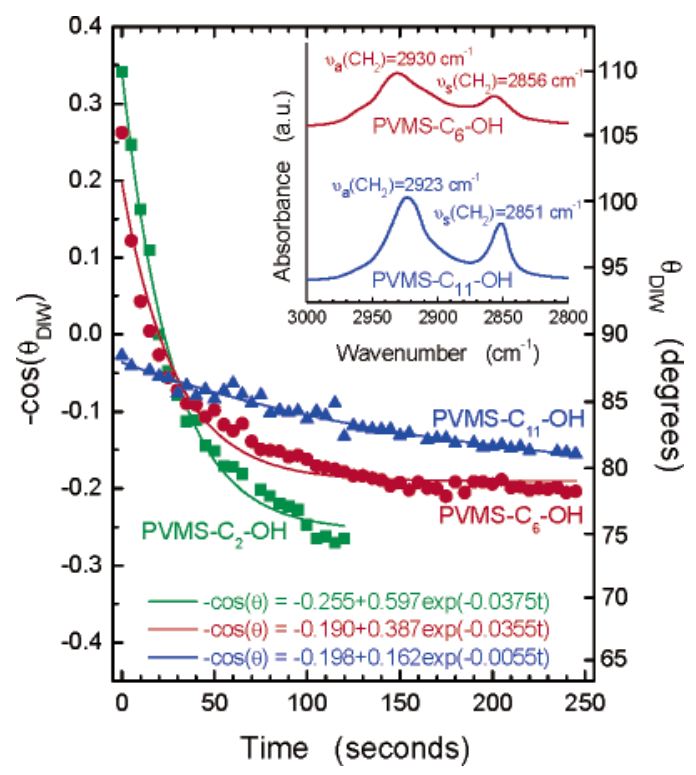

Figure 1. Time dependence of the deionized water wettabilities for PVMS$\mathrm{C}_{n}-\mathrm{OH}$ surfaces. Inset: ATR-FTIR for the PVMS $-\mathrm{C}_{6}-\mathrm{OH}$ and PVMS$\mathrm{C}_{11}-\mathrm{OH}$ substrates. The lower frequencies for the methylene asymmetric and symmetric stretches in PVMS $-\mathrm{C}_{11}-\mathrm{OH}$ are a signature of a semicrystalline surface. ${ }^{8-10}$ The error for $\theta_{\text {DIW }}$ is $\pm 1.5^{\circ}$.

PVMS $-\mathrm{C}_{2}-\mathrm{OH}$ exhibited a very fast drop in contact angle upon exposure to water $\left(\Delta \theta_{\text {DIW }} \approx 35^{\circ}\right)$. In contrast, PVMS $-\mathrm{C}_{11}-\mathrm{OH}$ showed only a modest change in contact angle $\left(\Delta \theta_{\text {DIW }} \approx 7^{\circ}\right)$. This sluggish response of PVMS $-\mathrm{C}_{11}-\mathrm{OH}$ upon exposure to water is attributed to a reduced mobility of surface-bound $-\mathrm{C}_{11}-$ side chains due to characteristic semicrystalline behavior. This behavior is inferred from a $\sim 10$-fold increase in $G^{\prime}$, a transition from a liquidlike to a semicrystalline-like state established by IR spectroscopy, and a transition from transparent to opaque state. In the inset to Figure 1, we plot the IR spectra of PVMS $-\mathrm{C}_{6}-\mathrm{OH}$ and PVMS$\mathrm{C}_{11}-\mathrm{OH}$ around the asymmetric and symmetric stretches of methylene units. The observed shifts in IR spectra provide information about the structure of the methylene linker. Specifically, while $-\mathrm{C}_{6}-$ adopts a liquid-like conformation, $-\mathrm{C}_{11}-$ exhibits a semicrystalline-like structure. ${ }^{8}$ These results are in accord with the findings of Allara and co-workers ${ }^{9}$ and those of Chaudhury and Owen. ${ }^{10}$

While the PVMS $-\mathrm{C}_{2}-\mathrm{OH}$ and $\mathrm{PVMS}-\mathrm{C}_{6}-\mathrm{OH}$ surfaces initially displayed fast surface reorientation kinetics after water exposure, it is the repeatability of the cycling behavior between wet and dry states that critically determines the viability of these surfaces as stimuli-responsive materials. We utilized a Cahn Dynamic Contact Analyzer (DCA) to measure wettability cycling with minimal sample handling. Each cycle consisted of placing a nitrogen-purged rectangular $\left(\sim 10 \times 10 \times 1 \mathrm{~mm}^{3}\right)$ PVMS slab 


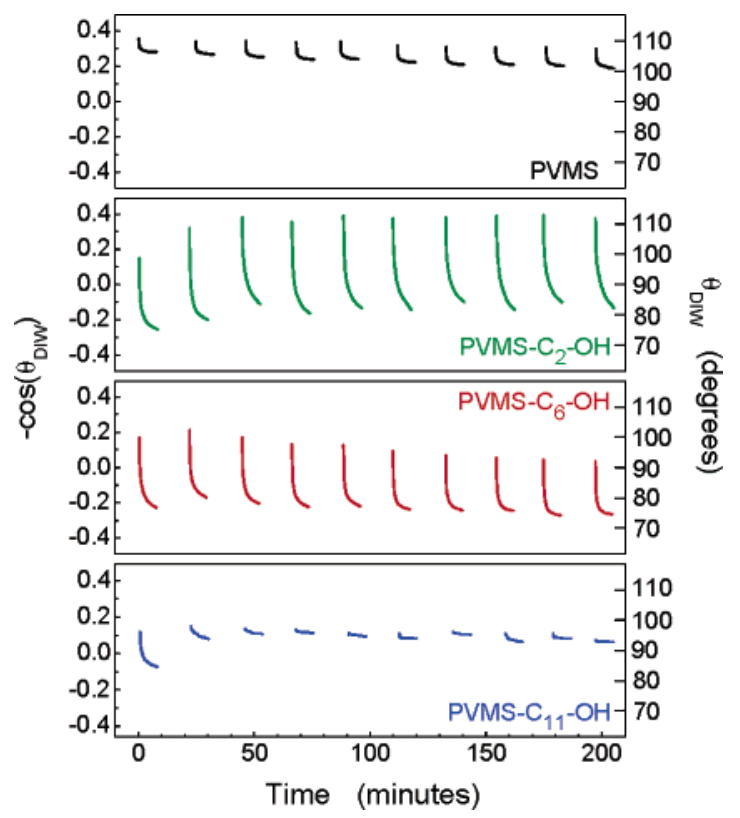

Figure 2. Dynamic contact angle of deionized water $\left(\theta_{\mathrm{DIW}}\right)$ as a function of time reported for 10 cycles on each $n$-alkane hydroxyl-terminated elastomer. For each $n$-alkane series, the data represent an average over three separate specimens. The error for $\theta_{\text {DIW }}$ is $\pm 3.8^{\circ}$.

above a beaker containing DI water at room temperature. The DCA stage was raised at a rate of $80 \mu \mathrm{m} / \mathrm{s}$ until the detection of the water-substrate interface. At that point, the stage was advanced for $3 \mathrm{~mm}$, held for $8.5 \mathrm{~min}$, and returned to its original position. The total water contact time was 9 min for each cycle. During each cycle, the DCA recorded the total force acting on the sample $\left(F_{\mathrm{t}}\right)$. $F_{\mathrm{t}}$ is related to contact angle through $F_{\mathrm{t}}=\mathrm{mg}+P \gamma_{\text {DIw }} \cos \theta_{\text {DIW }}+$ $V_{\mathrm{s}} \rho_{\text {DIW }} P$, where $\mathrm{mg}$ is the gravitational force zeroed out by taring the balance prior to the cycle run, $P$ is the wetted sample perimeter, $\gamma_{\text {DIW }}$ is the surface tension of DI water, $V_{\mathrm{s}}$ is the volume of the solid immersed in water, and $\rho_{\text {DIW }}$ is the DI water density. This technique facilitates measurement of advancing and receding contact angles as well as the change in contact angle during the sample hold time. Since the immersed volume of the sample remains constant during the hold time, only the wetting force $\left(P \gamma_{\text {DIw }} \cos \theta_{\text {DIW }}\right)$ changes as a function of time. After the completion of each cycle, the sample was blow-dried with nitrogen gas and another cycle was commenced. The lapse time between two consecutive cycles was $\sim 7 \mathrm{~min} ; 10$ wettability cycles were carried out for each sample.

Figure 2 depicts cycling wettabilities for unmodified PVMS and PVMS $-\mathrm{C}_{n}-\mathrm{OH}$; one cycle represents an average of measurements carried out on three separate specimens. For PVMS, the repeated oscillations displayed only minute changes in surface wettability, in agreement with previous findings. ${ }^{6,7}$ For PVMS $-\mathrm{C}_{6}-\mathrm{OH}$ and PVMS $-\mathrm{C}_{2}-\mathrm{OH}$, the repeated DCA oscillations demonstrate a close compliance with the static contact angle results. The difference between contact angles of dry and wet states for PVMS $-\mathrm{C}_{6}-\mathrm{OH}$ was consistent through all cycles with only a slight decrease in starting contact angle. PVMS $-\mathrm{C}_{2}-\mathrm{OH}$ cycles were even more repeatable with little sign of any dampening behavior. This substrate exhibited the largest change in contact angle and displayed a greater starting hydrophobic value after the initial cycle. Since this chain had the shortest methylene linker, the flexibility of the siloxane backbone remained intact, thus allowing for the restructuring to occur rapidly between the methyl and 3-mercaptopropanol substituents. The behavior of PVMS $-\mathrm{C}_{11}-\mathrm{OH}$ was more complex. While we observed an initial surface reorientation after the first water- dipping cycle, the surface "froze" in the hydrophobic state during all subsequent cycles. We attempted to recover the original hydrophilic response of PVMS $-\mathrm{C}_{11}-\mathrm{OH}$ by exposing the specimens to heated water $\left(95^{\circ} \mathrm{C}\right)$ for $60 \mathrm{~min}$. After the heat-treatment, the surface reorientation was frozen by immersion in $25^{\circ} \mathrm{C}$ water and drying with nitrogen. The surface reoriented to a stable, more hydrophilic surface $\left(\theta \approx 80^{\circ}\right)$. Continued monitoring of the specimen revealed a slow reconstruction to the hydrophobic state $\left(\theta \approx 96^{\circ}\right)$ after exposure to air for several days. We note that it is possible to quickly restructure the surface to a starting contact angle of $\sim 105^{\circ}$ if the sample is heated in water and allowed to slowly air-cool in air versus quenching in $25{ }^{\circ} \mathrm{C}$ water. This result is consistent with the findings of Carey ${ }^{12}$ and Holmesfarley ${ }^{13}$ for surface restructuring of oxidized polybutadiene and oxidized polyethylene, respectively. These results along with the IR data presented in Figure 1 indicate that after the first cycle, $-\mathrm{C}_{11}-\mathrm{OH}$ groups were "trapped" just beneath the surface in a semicrystalline structure, formed presumably via interactions between neighboring $-\mathrm{C}_{11}-$ chains, and did not respond to the presence of water on the surface. Annealing the sample appeared to partially "melt" the semicrystalline regions as the substrate became transparent, hence allowing the mercaptododecanol side chains to moderately reconstruct at the surface.

We summarize by emphasizing several key points of the novel design of our surfaces. They represent the only polymeric system we are aware of that responds almost instantaneously to wettability changes at room temperature $\left(\Delta \theta \approx 2 \%\right.$ for PVMS $\left.-\mathrm{C}_{2}-\mathrm{OH}\right)$. This behavior results from the high flexibility of the siloxane backbone and the large disparity of surface energies between the hydrophobic methyl and the hydrophilic mercaptoalkanol side chains. The response rate depends on the number of methylene spacers in the mercaptoalkanol. While for $n=2$ and 6 , the surface reconstructs almost instantaneously, $n=11$ specimens resist reconstruction because of strong van der Waals forces, leading to the formation of semicrystalline regions. Finally, we have shown that liquid-like siloxane surfaces ( $n=2$ and 6$)$ can be continually oscillated between polar and nonpolar states for at least 10 cycles, with no loss of wettability for the 3-mercaptopropanol-modified substrate.

Acknowledgment. The generous financial support from the Office of Naval Research is appreciated. We thank Stefan Franzen and Simon Lappi for the use of the ATR-FTIR.

\section{References}

(1) Russell, T. P. Science 2002, 297, 964-967.

(2) Kasemo, B. Surf. Sci. 2002, 500, 656-677.

(3) Koberstein, J. T. J. Polym. Sci. B, Polym. Phys. 2004, 42, 2942-2956.

(4) Khongtong, S.; Ferguson, G. S. J. Am. Chem. Soc. 2002, 124, 72547255.

(5) Sun, T.; Wang, G.; Feng, L.; Liu, B.; Ma, Y.; Jiang, L.; Zhu, D. Angew. Chem., Int. Ed. 2004, 43, 357-360.

(6) Efimenko, K.; Crowe, J. A.; Manias, E.; Schwark, D. W.; Fischer, D. A.; Genzer, J. Polymer 2005, 46, 9329-9341.

(7) Crowe, J. A.; Efimenko, K.; Genzer, J.; Schwark, D. W. In Responsive Polymer Materials: Design and Applications; Minko, S., Ed.; Blackwell Publishing: Cambridge, MA, 2006; Chapter 11, pp 184-205.

(8) Snyder, R. G.; Strauss, H. L.; Elliger, C. A. J. Phys. Chem. 1982, 86, 5145-5150.

(9) Allara, D. L.; Parikh, A. N.; Judge, E. J. Chem. Phys. 1994, 100, 17611764.

(10) Chaudhury, M. K.; Owen, M. J. J. Phys. Chem. 1993, 97, 5722-5726.

(11) Rozga Wijas, K.; Chojnowski, J.; Boileau, S. J. Polym. Sci. A, Polym. Chem. 1997, 35, 879-888.

(12) Carey, D. H.; Ferguson, G. S. Macromolecules 1994, 27, 7254-7266.

(13) Holmesfarley, S. R.; Reamey, R. H.; Nuzzo, R.; McCarthy, T. J.; Whitesides, G. M. Langmuir 1987, 3, 799-815.

JA056926U 